\title{
Behçet's disease leaves the silk road
}

\author{
Anna Woźniacka ${ }^{1}$, Piotr Jurowski², Anastazy Omulecki ${ }^{1}$, Marek Kot ${ }^{1}$, Bożena Dziankowska-Bartkowiak ${ }^{1}$
}

1Department of Dermatology and Venereology, Medical University of Lodz, Lodz, Poland

Head of the Department: Prof. Anna Woźniacka MD, PhD

2Department of Ophthalmology and Visual Rehabilitation, Military Medical University of Lodz, Lodz, Poland

Head of the Department: Prof. Piotr Jurowski MD, PhD

Postep Derm Alergol 2014; XXXI, 6: 417-420

DOI: 10.5114/pdia.2014.40943

\begin{abstract}
Behçet's disease is rarely recognized among Caucasians, while it is endemic in countries of the Eastern and Central Asia, especially in Turkey. The aim of the manuscript is to present the clinical picture, diagnosis criteria and therapeutic approaches of this rare disease which, because of intensive migration, can occur not only in particular regions of the world.
\end{abstract}

Key words: Behçet's disease, vasculitis.

\section{Introduction}

Behçet's disease (BD) was probably first described by Hippocrates in the $5^{\text {th }}$ century BC [1]. However, it bears the name of its modern explorers: Benedictos Adamantiades (Greek ophthalmologist) and Hulusi Behçet (Turkish dermatologist) [2, 3]. The disease is predominantly diagnosed in the Far and Middle East countries, mostly in Turkey, where its incidence is evaluated as 380 cases per 100,000 persons in the population. Definitely less cases are recognized in Japan (100/100 000), Europe (1/300 000) and the USA (1-2/100 000). The etiology of the disease is not fully understood. A lot of scientific works indicate that the development of changes occurs in genetically predisposed people, in which infectious agents, probably viral, contribute to the launch of the dysregulation of the immune response, and then the development of inflammatory changes of the blood vessels. In Poland, only a few cases of this disease have been described so far.

\section{Case report}

A 23-year-old student, single, was admitted to the Department of Dermatology of the Medical University of Lodz, due to unresponsive treatment of the recurrent and painful ulcers localized on the mucosa of the mouth and on the skin of the scrotum. He has been treated unsuccessfully for 4 years with topical corticosteroids and antibiotics in his local Medical Center. He reported pain and diminished flexibility of knee joints and temporary presence of small edematous papules which used to ap- pear the next day after topical injury (positive pathergic reaction). He has never been to Asia or Turkey.

In 2008, the first symptoms of anterior and intermediate uveitis of both eyes appeared, so the patient was admitted to the Department of Ophthalmology of the regional hospital where local and systemic steroid and mydriatic therapy was introduced. Despite treatment, diminishing of visual acuity was observed. The first clinical evaluation in the Department of Dermatology was performed in June 2011. Thorough medical examination revealed an annular ulcer localized on the upper lip mucosa, $0.7 \mathrm{~cm}$ of diameter, with smooth edges and clinical symptoms of inflammation on the perimeter. The bottom was covered with yellowish coating (Figure 1). A similar lesion, diameter of $1 \mathrm{~cm}$, was present on the scrotum (Figure 2). For the first time, BD was diagnosed and treatment with colchicine in a dose of $2 \mathrm{mg} /$ day was started. Since then, dermatologists have collaborated with the ophthalmologist who reported episcleral hyperemia as well as inflammatory reaction within the anterior chamber and vitreous body. In the course of the aforementioned treatment, exudates diminished and good visual acuity was restored. Although, initially this therapy brought also the remission of the mucosal lesions, but after 2 months it came to the severity and uveitis also recurred (Figure 3). A subsequent drop of visual acuity, pain and photophobia was reported. At that time, the visual acuity was 0.05 in the right and $0.32 \mathrm{cc}$ $-2.5 \mathrm{dsph} / 1.0$ ax 175 in the left eye. The dense anterior and vitreous protein and cellular reaction was observed.

Address for correspondence: Prof. Anna Woźniacka MD, PhD, Department of Dermatology and Venereology, Medical University of Lodz, 5 Krzemieniecka St, 94-017 Lodz, Poland, phone: +48 4268625 70, e-mail: wozniacka@bmp.net.pl Received: 9.10.2013, accepted: 29.10.2013. 


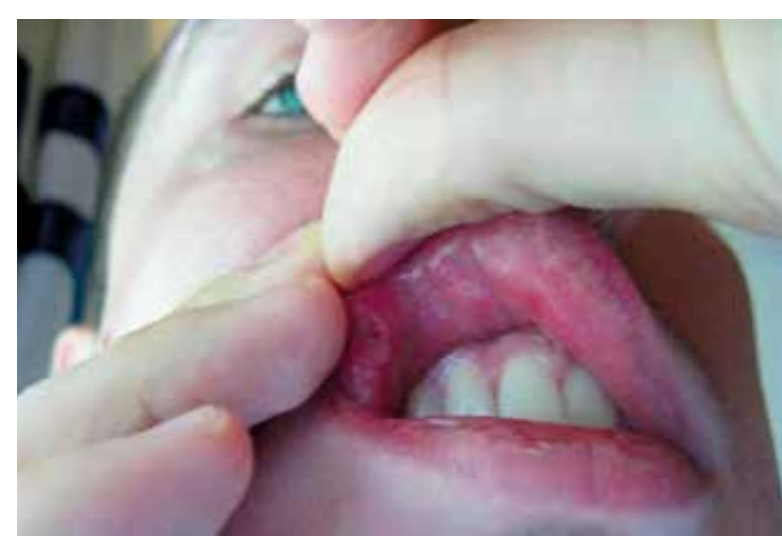

Figure 1. Annular ulcer localized on the upper lip mucosa, $0.7 \mathrm{~cm}$ of diameter, with smooth edges and clinical symptoms of inflammation on the perimeter

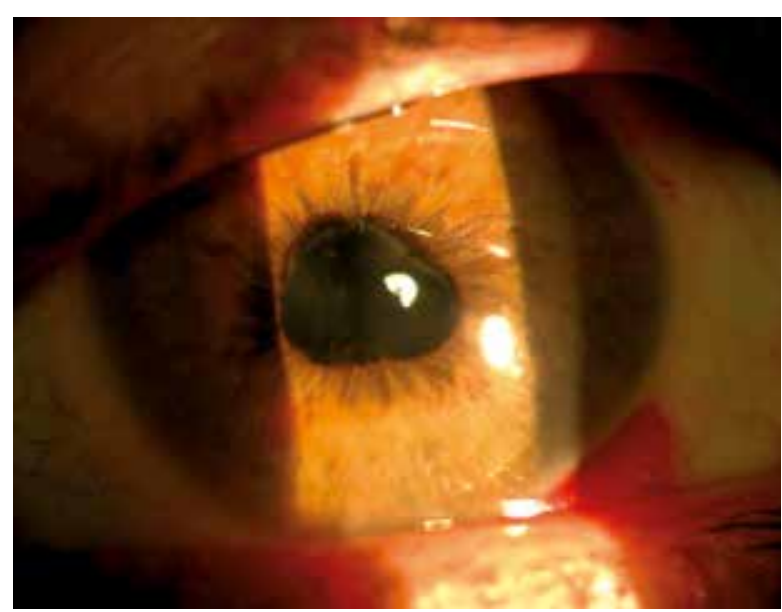

Figure 3. Anterior segment photography of the right eye. Posterior synechia, ovalization of the pupil and pigment on the lens surface are seen

There were posterior capsular cataract and intense posterior synechia, and fibrotic bands on the vitreous base in the right eye (Figure 4). Intraocular pressure increased up to $30 \mathrm{~mm} \mathrm{Hg}$.

For this reason, phacoemulsification and pars plana vitrectomy with silicone oil tamponade of the right eye was provided. Methylprednisolone in a dose of $24 \mathrm{mg}$ daily was introduced. As this kind of treatment was insufficient, azathioprine in the initial dose of $100 \mathrm{mg} /$ day was added. Due to uveitic process not responding, the azathioprine dose was increased up to $200 \mathrm{mg} /$ day with concomitant methylprednisolone of $24 \mathrm{mg} /$ day. Additionally, peribulbar, intravitreal triamcinolone injection and topical intraocular pressure (IOP) lowering agents were introduced. This increased dose of azathioprine and combined treatment with immunosuppressive agents brought clinical alleviation. Visual acuity slowly but systematically improved and finally the patient achieved 0.2 of best corrected visual acuity. Fundus photography

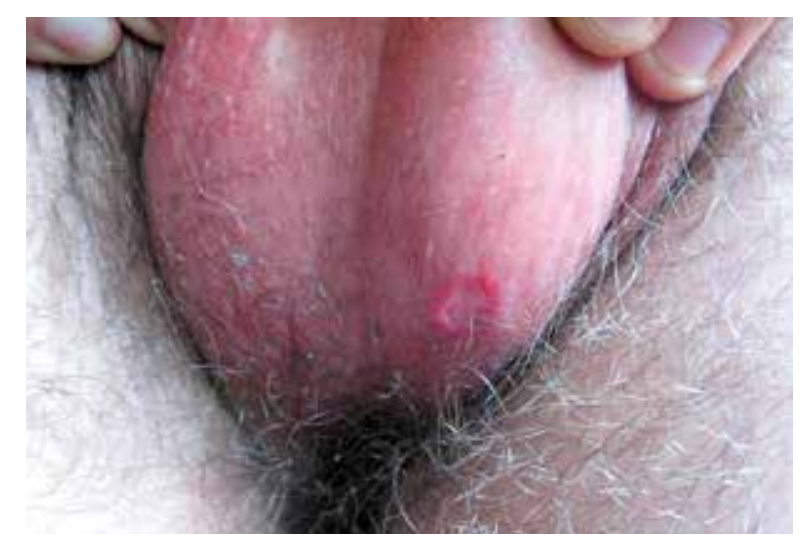

Figure 2. Annular ulcer, diameter of $1 \mathrm{~cm}$ on the scrotum

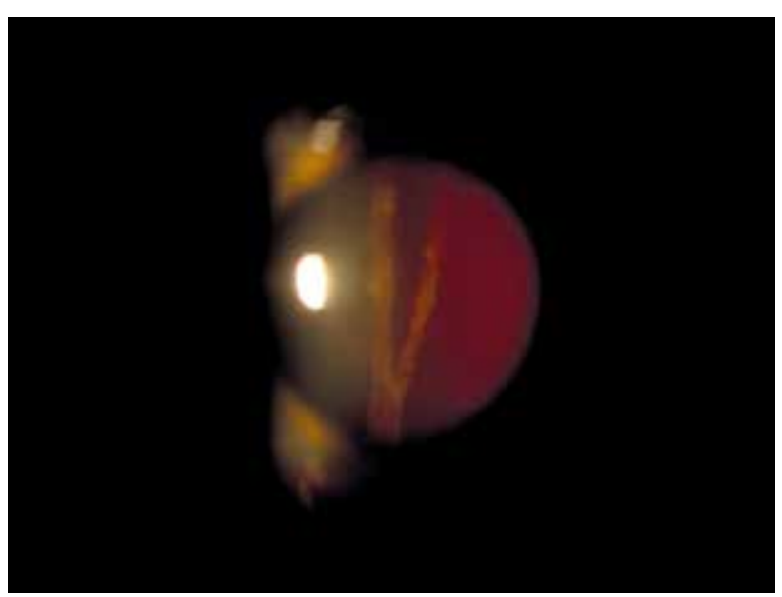

Figure 4. Band-like exudates extending from the vitreous body base in both eyes were observed

and fluorescein angiography revealed some pigmentary changes within fovea corresponding with retinal pigment epithelium and photoreceptors layer alterations observed in SOCT scans (Figures 5, 6). Laboratory tests (ASO, OB, complete blood count, urinalysis, urine culture, biochemical tests: blood glucose, creatinine, urea, potassium, sodium, bilirubin, iron) did not reveal any abnormalities. However, patient reported tonsils excess, on the swab taken from the throat only saprophytic microorganisms were detected. Waller-Rose test and latex RF were negative. Radiological chest examination and nasal sinus revealed normal status. Ultrasound examination of the abdomen and the heart did not reveal any deviations from the normal status.

Behçet's disease was diagnosed on the basis of the clinical picture, i.e. recurrent aphthous ulcers in the mouth, sores in the genital area, symptom of pathergia and concomitant uveitis. In addition, genetic testing confirmed the HLA-B51, which is considerably more common 


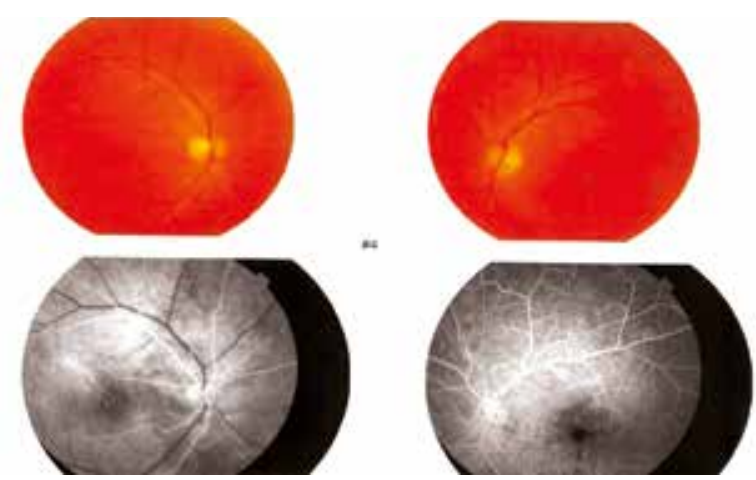

Figure 5. Fundus color photography and fluorescein angiography during the venous phase. Multiple pigmentary changes within macula of both eyes are seen

in this group of patients and supported the clinical conclusion.

\section{Discussion}

Behçet's disease used to be a rare condition among Polish inhabitants. The growing number of cases of this rare disorder makes it necessary to remind dermatologists about the possibility of the occurrence of BD also among Caucasians living in regions other than Asia [4, 5].

However, the cause of BD remains unknown, epidemiologic findings suggest that the autoimmune process is triggered by an infectious or environmental agent, specific to the geographic region. However, there is no information supporting the role of a single microorganism as a specific cause of autoimmune dysregulation. The infectious model is supported by the observation that oral ulcers precede the establishment of the disease by months or years and disease relapses are frequent.

The genetic susceptibility is strongly associated with the presence of the HLA-B51 allele, with a stronger association in Turkish and Japanese patients in comparison to Caucasians [6, 7]. The unusual geographic distribution of the disease and this high association with HLA-B51, as in our patient, may be the strongest indicator that certain genes are directly responsible for $\mathrm{BD}$.

As there are no specific diagnostic laboratory tests or histopathologic findings which confirm the supposition, the final diagnosis must be based on clinical criteria. Clinical presentation is heterogeneous, and not all symptoms appear simultaneously. Evolution of the disease varies due to ethnic, geographical and individual differences. Oral aphtae are usually the first manifestation of BD and like in our patient precede by many years the onset of other manifestations.

Although $\mathrm{BD}$ is a relatively new entity (described in 1937) it has many sets of classification criteria. The most important ones were created in 1990 by the International Study Group (ISG). The ISG criteria use 5 items. Two items are mucous membrane manifestations (oral aph-

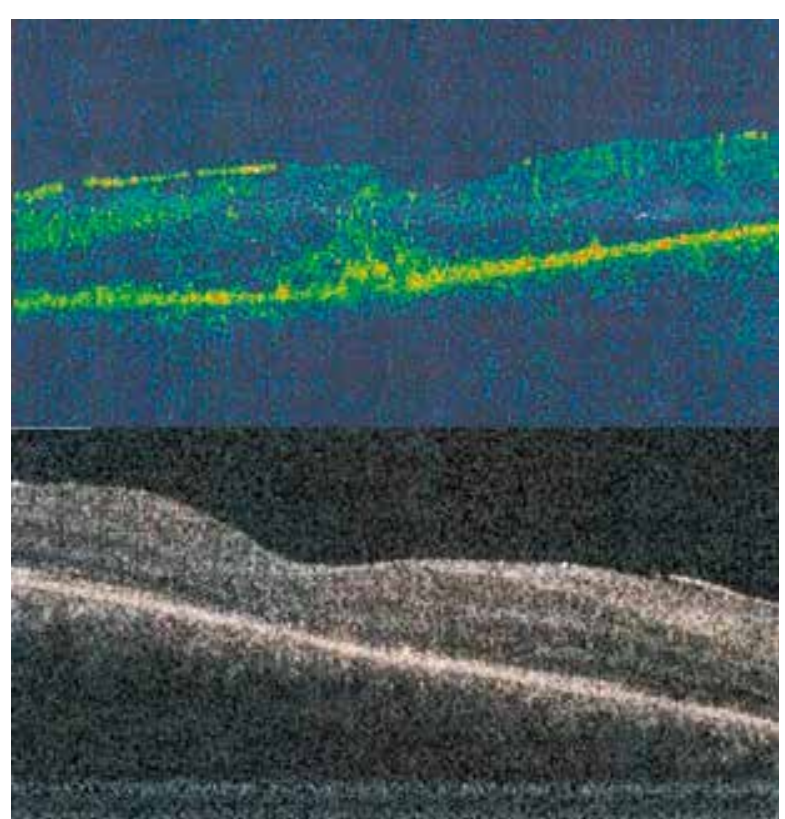

Figure 6. Abnormalities of retinal pigment epithelium and photoreceptors layer in the fovea of the left eye and epiretinal membrane on the retinal surface are seen in SOCT scans

thosis and genital aphthosis), the third item is skin manifestations, like pseudofolliculitis and erythema nodosum. The fourth item is ocular manifestations (anterior uveitis, posterior uveitis or retinal vasculitis). The fifth item is the presence of the pathergy phenomenon. In ISG criteria the presence of oral aphthosis is obligatory, and two others out of the remaining four are necessary to establish the diagnosis. In the presented case recurrent aphthosis in both localizations was observed. Moreover, the patient had ocular manifestations and the pathergy phenomenon, what made the diagnosis stronger.

For more than 16 years the ISG criteria have been the most used criteria for diagnosing BD, however the majority of validation studies failed. Therefore, in 2006, the International Team for the Revision of the International Criteria for Behçet's Disease (ICBD), gathered to establish best accuracy and optimum sensitivity and specificity and created a new set of diagnostic criteria (ICBD) [8]. Vascular manifestations like superficial phlebitis, deep vein thrombosis, large vein thrombosis, arterial thrombosis and aneurysm were added. In the ICBD, genital aphthous lesions and eye lesions have a greater diagnostic value than the others, so they get 2 points each. The other 4 items get one point. To classify the patient as having BD three points or more are necessary. In our patient, recurrent oral and genital ulcerations, ocular inflammatory changes and positive pathergy test were observed, so according to the ICBD scale, he has achieved 6 points. As the disease is very rare in the Central Europe, additional genetic studies were performed to support the diagnosis. 
It is generally accepted that multiorgan system involvement often begins with mucocutaneous involvement. In the presented patient, besides the skin, only one systemic symptom of the disease, i.e. ocular involvement was diagnosed. Organ-specific manifestations are characterized by exacerbations and relapsing course. Ocular involvement is probably the most frequent (28-80\%) and is an important cause of morbidity. This localization is more frequent in males than in females and usually males tend to have a worse visual prognosis.

Ocular involvement in BD comprises a wide spectrum of symptoms. In most cases, the ocular symptoms follow the oral and genital ulcers by 3-4 years, however ocular disease is the initial manifestation in about 20\% of cases. Initially, symptoms include periorbital pain, redness, photophobia, and blurred vision. Behçet's disease is characterized by severe recurrent attacks of intraocular inflammation, i.e. episcleritis, scleritis, keratitis and anterior uveitis, posterior uveitis or panuveitis. Vitritis with large exudates due to vasculitis can lead to macular edema. Optic neuritis followed by optic atrophy and exudates, retinal detachment are the reasons for visual loss in some cases. As a consequence of intermediate uveitis, the posterior capsular cataract can occur [9]. In our case, phacoemulsification with artificial intraocular lens implantation combined with pars plana vitrectomy and silicone oil tamponade with the aforementioned medical treatment stabilized the posterior segment of the eye allowing for slow but systematic visual improvement.

Among other organs, arthritis and arthralgia are most often affected and observed in as many as $60 \%$ of patients. Mainly the lower extremities are affected, especially the knee like in our patient, but ankles, wrists and elbows can also be involved.

Current treatment of BD involves symptomatic relief with prevention of relapse. Treatment is largely empirical since well-controlled studies are difficult to conduct due to the heterogeneity of the disease, and the unpredictable course with exacerbation and remission. Conventional therapeutic approaches include nonsteroidal anti-inflammatory agents, corticosteroids with or without other immunosuppressive drugs, such as cyclophosphamide, azathioprine, sulfasalazine, colchicine, methotrexate or cyclosporine A.

However, nowadays new insights into BD immunopathogenesis have led to novel therapeutic approaches, mainly off-label use of antitumor necrosis factor agents though old strategies are still effective [10]. In the literature, there are many reports underlining good clinical results after combined treatment with azathioprine and corticosteroids. Several authors describe patients with $\mathrm{BD}$ in whom multiple pulmonary artery aneurysms were completely resolved after combined treatment with corticosteroids and azathioprine [11, 12]. In our patient, a good clinical effect and lack of recurrence in 1 year's follow-up force this way of a therapeutic approach.

\section{Acknowledgments}

This study was supported by a grant for scientific purposes (No. 503/1-152-01/503-01) from the Medical University of Lodz, Poland. The authors report no conflict of interest.

\section{References}

1. Altenburg A, Papoutsis N, Orawa H, et al. Epidemiology and clinical manifestations of adamantiades-Behcet disease in Germany - current pathogenetic concepts and therapeutic possibilities. J Dtsch Dermatol Ges 2006; 4: 49-66.

2. Zouboulis CC. Benediktos Adamantiades and his forgotten contributions to medicine. Eur I Dermatol 2002; 12: 471-4.

3. Ustun C. A famous Turkish dermatologist, Dr Hulusi Behcet. Eur J Dermatol 2002; 12: 469-70.

4. Jakubowicz O, Żaba R, Czarnecka-Operacz M, Silny W. Suspicion of Behçet's disease - case report. Postep Derm Alergol 2009; 3: 155-60.

5. Romańska-Gocka K, Gocki J, Placek W, et al. Behçet disease case report and review. Postep Derm Alergol 2009; 4: 224-8.

6. Miauki N, Ota M, Yabuki K et al. Localization of the pathogenic gene of Behcet's disease by microsatellite analysis of three different populations. Invest Ophtalmol Vis Sci 2000; 41: 3702-8.

7. Maldini C, Lavalley MP, Cheminant M, et al. Relationships of HLA-B51 or B5 genotype with Behcet's disease clinical characteristics: systemic review and meta-analyses of observational studies. Rheumatology (Oxford) 2012; 51: 887-900.

8. di Meo N, Bergamo S, Vidimari P, et al. Analysis of diagnostic criteria in adamantiades-Behçet disease: a retrospective study. Indian J Dermatol 2013; 58: 275-7.

9. Kaçmaz RO, Kempen JH, Newcomb C, et al. Ocular inflammation in Behçet disease: incidence of ocular complications and of loss of visual acuity. Am J Ophthalmol 2008; 146: 828-36.

10. Köse O. Development of immunopathogenesis strategies to treat Behçet's disease. Patholog Res Int 2012; 2012: 261989.

11. Ben Dhaou B, Ketari S, Boussema F, et al. Pulmonary aneurysms in Behçet's disease completely resolved after medical therapy. Rev Pneumol Clin 2012; 68: 311-4.

12. Agha A, Bella AM, Assiri AH, et al. Can Behçet's disease related pulmonary arterial aneurysms be completely resolved? Open Rheumatol J 2011; 5: 88-90. 